\title{
CONTEXTOS DE USO DE LAS PLANTAS VINCULADAS al Complejo Pica TARApacÁ, Andes Centro- SUR: ARQUEOBOTÁNICA Y AGRICULTURA EN EL PERÍODO INTERMEDIO TARDÍO (CA. 1250-1450 DC)
}

\author{
Magdalena García B. ${ }^{1}$ y Mauricio Uribe R. ${ }^{2}$
}

\section{* InTROducción}

Este artículo da a conocer los macrorrestos vegetales obtenidos en los asentamientos de Nama, Camiña y Jamajuga, ubicados en la precordillera de Tarapacá, Norte Grande de Chile (19 ${ }^{\circ}$ lat. S). El propósito principal es aportar a la documentación del Complejo Pica Tarapacá durante la fase Camiña (ca. 1250-1450 DC), a través de la evaluación de los distintos contextos en que las plantas fueron utilizadas al interior del espacio habitacional. Las muestras provienen de la excavación de 28 recintos y fueron recuperadas tanto de harnero como por flotación. El análisis permitió definir al menos dos contextos de uso de las plantas, uno que interpretamos como doméstico o familiar y otro más bien público o ceremonial. Ambos conformarían ámbitos diferentes y complementarios dentro de la dinámica cotidiana de los agricultores tarapaqueños durante el período Intermedio Tardío.

Palabras claves: maíz - quínoa - espacio domestico - Complejo Cultural Pica Tarapacá.

This paper describes the plant remains from the archaeological sites of Nama, Camiña and Jamajuga, located in the valley and highlands of Tarapacá, northern Chile ( $19^{\circ}$ lat. S). The main purpose of this paper is to provide additional botanical information regarding the Pica Tarapacá Complex during the Camiña archaeological phase ( $c a$. 1250-1450 AD), through the analysis of plants from ancient household spaces. The sample comes from 28 excavation units and were obtained directly from the sieve and flotation. We defined at least two contexts

associated to plant use. The first one is associated to a private and domestic sphere, while the other corresponds to the use of plants in open public and ceremonial contexts. Both practices are defined as different but complementary within the daily lives of the Tarapacá farmers during the Late Intermediate Period.

Key words: maize - quinoa - household space - Pica Tarapacá Cultural Complex.

Recibido: julio 2011. Aceptado: marzo 2012.
Desde la antropología, distintos autores han señalado que los principios fundamentales de la organización socioeconómica de las comunidades andinas son la reciprocidad, la redistribución y el intercambio. La reciprocidad se sustentaba tradicionalmente en la posesión comunitaria de la tierra, la cual se trabajaba colectivamente con una marcada orientación agrícola y ganadera. Los terrenos productivos eran asignados y regulados de acuerdo al sistema de parentesco y su repartición a nivel familiar se iniciaba con el matrimonio (Alberti y Mayer 1974; Murra 1975).

Esta forma de organización hunde sus raíces en tiempos prehispánicos (Schiappacasse et al. 1989; Castro 2002). En épocas inmediatamente previas a la llegada de los incas (período Intermedio Tardío, ca. 1000-1450 DC), las comunidades agrícolas del área centro-sur andina habitaron en asentamientos de gran tamaño, construidos en altura, los cuales reflejan una alta densidad poblacional y promueven una organización política segmentaria (Uribe 2006; Adán et al. 2007; Acuto 2007). Estos poblados se encuentran conectados directamente a extensas áreas edificadas para el cultivo tanto en laderas como en fondos de valle, las cuales conforman sistemas agrícolas diferenciados, con riego artificial manejado desde vertientes o el curso principal de las quebradas donde estos se emplazan. Su tecnología constructiva es similar a la de los poblados, basadas en la lógica de aterrazamiento

\footnotetext{
1 Programa de Doctorado en Antropología, Universidad Católica del Norte. Becaria CONICYT. Centro de Investigación del Hombre en el Desierto (CHIDE), Arica. Camino El Bajo 160, El Canelo, San José de Maipo, CHILE. Email: manegarciab@yahoo.com

2 Departamento de Antropología, Facultad de Ciencias Sociales, Universidad de Chile. Ignacio Carrera Pinto 1045, Ñuñoa, Santiago, CHILE. Email:mur@uchile.cl
} 
y contención del terreno. Dichos andenes, en definitiva, permitieron crear microclimas que favorecen la reproducción exitosa de cultivos semitropicales, como el maíz, fuera de sus ámbitos de origen y constituyen un elemento diagnóstico del proceso de intensificación que sufrió la agricultura antes de la llegada del Inca (Murra 1980; Castro 1988; Adán et al. 2007; Albeck 2011).

En la precordillera de Arica y Tarapacá (2400-3300 m.s.n.m.), los altos rendimientos productivos sustentaron la intensificación y ampliación de las redes de interacción, principalmente con el altiplano de Carangas, Quillacas y Lípez, vínculos que se expresan en la incorporación de rasgos altiplánicos a la arquitectura local (cistas, chullpas) así como a la cerámica (tipos Chilpe, Taltape), entre otros (Muñoz y Chacama 2006; Uribe 2006).

Junto a esta infraestructura productiva, la intensificación de la agricultura se manifiesta al interior de los poblados a partir de múltiples estructuras de almacenaje subterráneo o collcas, así como en los restos vegetales que se han preservado. Las primeras se ubican al interior de las viviendas y en patios comunes o plazas, por lo que han sido vinculadas con el almacenaje familiar y comunitario, respectivamente. Los productos almacenados, especialmente el maíz, debieron asegurar la subsistencia familiar, la redistribución comunitaria y la interacción con comunidades foráneas (Muñoz y Chacama 2006; Adán et al. 2007). Por último, los restos vegetales si bien son generalmente vulnerables al paso del tiempo, corresponden a las evidencias concretas de lo almacenado y consumido en estos contextos.

Nuestro trabajo aborda esta última línea de evidencia. En este sentido, se darán a conocer los restos vegetales recuperados en los asentamientos Camiña, Nama y Jamajuga, precordillera de Tarapacá, Norte Grande de Chile, con el fin de documentar los contextos de uso de las plantas al interior del espacio habitacional. Para ello, llevaremos a cabo, en primer lugar, una evaluación diacrónica de la agricultura prehispánica en la región, fundamentalmente en relación a los valles bajos y la pampa del Tamarugal. Posteriormente, las evidencias obtenidas se analizarán cualitativa y cuantitativamente y se contextualizarán en relación al resto de las materialidades que conforman los contextos arqueológicos. Finalmente, los datos obtenidos se discutirán en función de la problemática planteada.

\section{* Manejo de las plantas durante el primer MILENIO DE NUESTRA ERA}

La región cultural de Tarapacá comprende un amplio espacio que abarca desde la quebrada de Camiña o Tana y el altiplano de Isluga por el norte, hasta el río Loa por el sur, entre la costa del océano Pacífico y la puna. Colinda por el norte con la región de Arica y por el sur con Atacama, vinculando así los Valles Occidentales con la Circumpuna (Figura 1). Entre las cuencas mencionadas, la cordillera de la Costa emerge como una barrera que impide el paso al mar de las decenas de quebradas que descienden serpenteantes desde la puna, destacando Aroma, Tarapacá, Maní y Guatacondo, las cuales desaguan esporádicamente en la pampa alimentando las napas subterráneas que permitieron sustentar enormes extensiones de bosques de tamarugos (Prosopis tamarugo) y algarrobos (Prosopis chilensis, P. flexuosa, P. alba, P. strombulifera y P. burkartii). De allí su nombre Pampa del Tamarugal (Briner 1985; Niemeyer 1989).

De esta manera, bosques, quebradas y oasis (p.e., Pica) generan un alto contraste en medio del desierto absoluto y representan, al mismo tiempo, ejes de organización del asentamiento humano desde tiempos prehispánicos, desde donde se desarrollaron diversos y particulares mecanismos de complementariedad económica con otros pisos altitudinales y ecológicos (Núñez 1965, 1979; Schiappacasse et al. 1989; Moragas 1995).

En Tarapacá, la mayor parte de los antecedentes arqueobotánicos se concentran en las tierras bajas donde existe una muy buena preservación de los restos vegetales. En los sitios vinculados al período Formativo (ca. 900 AC950 DC), la pampa del Tamarugal aparece como un verdadero refugio para quienes se movilizaban entre la costa y las tierras altas; la estabilidad propiciada por la oferta exuberante de vainas de algarrobo sería definitoria para la formación económico social de estas poblaciones, generando las condiciones necesarias para el surgimiento de la vida aldeana, la agricultura y, en definitiva, la complejidad social (Núñez 1974, 1979, 1982). Siguiendo esta visión economicista, aunque no por ello menos perspicaz, el reflejo de este proceso se encontraría en la edificación de las primeras aldeas agrícolas y sedentarias, excepcionalmente elocuente en Caserones, emplazado adyacente a los bosques de Prosopis ps. de la pampa (Núñez 1966, 


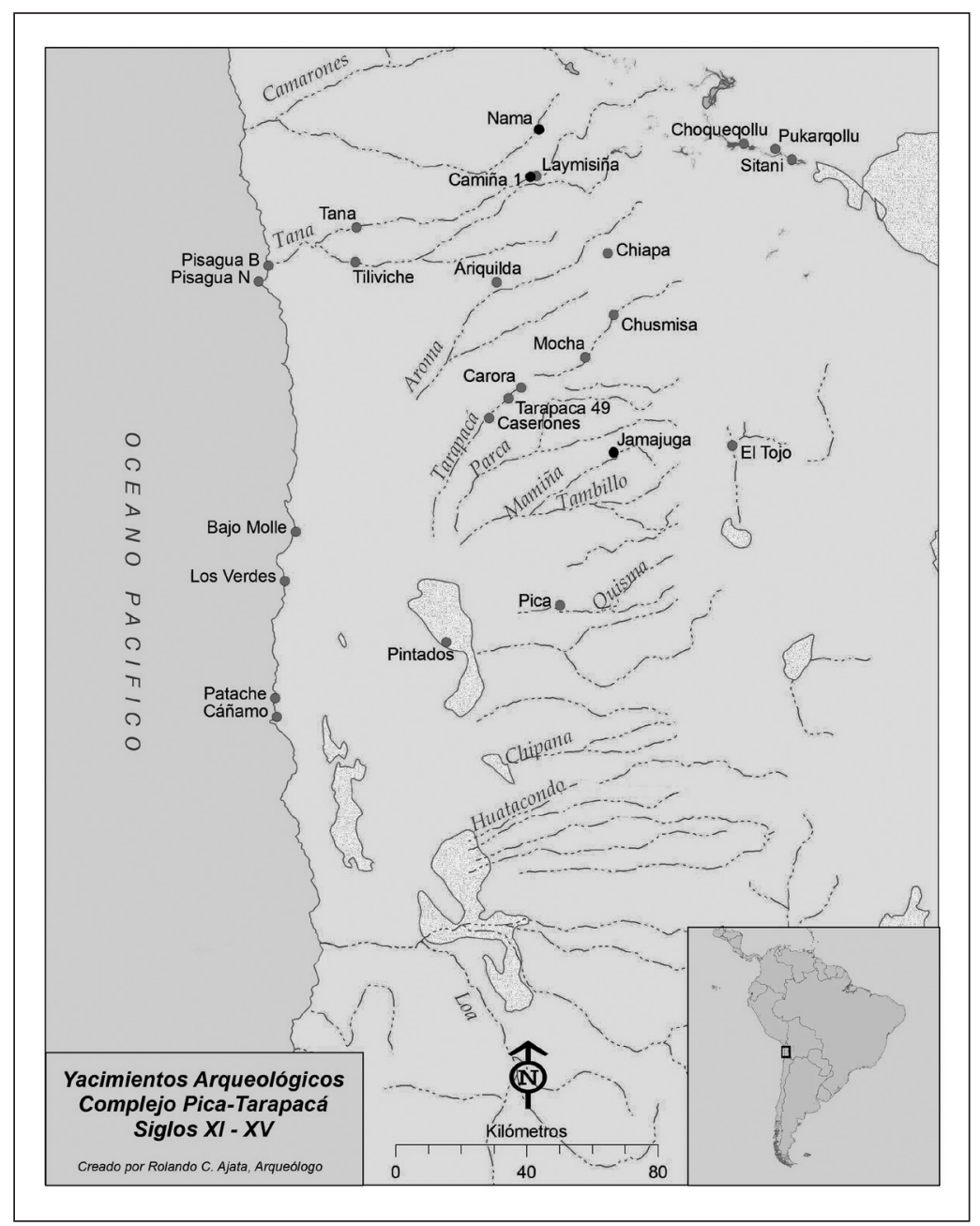

Figura 1. Principales asentamientos asociados al Complejo Pica Tarapacá, destacando en negro los sitios estudiados.

1982). En más de 700 años de ocupación, esta aldea manifiesta una importante densificación de los sectores habitacionales y de estructuras orientadas al almacenaje, lo cual estaría relacionado con una sobreproducción alimentaria enfocada al consumo e intercambio fundamentalmente con la costa (True 1980; Núñez 1982). Entre los cultivos mencionados por los autores, se encuentra maíz (Zea mays), poroto (Phaseolus vulgaris), pallar (Phaseolus lunatus), zapallo (Cucurbita sp.), papa (Solanum tuberosum), quínoa (Chenopodium quinoa), maní (Arachis hypogaea), ají y algodón (no se mencionan nombres científicos). Entre las especies silvestres, señalan algarrobo (Prosopis chilensis), totora (Scirpus sp.), tamarugo (Prosopis sp.), molle (Schinus molle) y pacay (Inga feuilleu) (Núñez 1966, 1979, 1982).
Al trabajo realizado por Núñez, se suman los resultados obtenidos recientemente que corroboran una ocupación hasta la primera mitad del período Intermedio Tardío o fase Tarapacá (ca. 950-1250 DC), adjudicada al Complejo Pica Tarapacá (Uribe et al. 2007). En relación a los restos vegetales, se registró un notable protagonismo de vainas y semillas de algarrobo (Prosopis sp.) que saturaban los depósitos. En menor medida, se hallaron frutos de molle (Schinus molle) y chañar (Geoffroea decorticans), todos los cuales vienen a documentar la gran importancia alimenticia, constructiva, artesanal y como combustible de los árboles de la pampa durante la vigencia del asentamiento. Fueron muy recurrentes también las camadas de vegetales constituidas por tallos de brea (Tessaria absinthioides) y 
cañaveral (Phragmites australis), conformando verdaderos estratos que, en ciertos casos, corresponden a techumbres colapsadas. Asimismo, la recurrencia de semillas de algodón (Gossypium sp.) se interpretó como parte de las impurezas descartadas durante el proceso de limpieza de las fibras para su posterior hilado, fortaleciendo la idea de una obtención local. Por último, se identificaron especies de la vertiente oriental tales como maní (Arachis hypogaea), una especie foránea de algarrobo (Prosopis algarrobilla) utilizada como materia prima de un artefacto expeditivo y una semilla de cebil (Anandenanthera sp.), constituyéndose esta última en la primera evidencia directa de psicoactivos al interior de basuras domésticas en el Norte Grande (García et al. 2012).

Por otra parte, en el cementerio contemporáneo Tarapacá 40, frente a Caserones, los restos vegetales reiteran una tradición de agricultura temprana vinculada a actividades intensivas de recolección de algarrobo (Prosopis sp.). Se documenta la consolidación de una sociedad agraria consumidora de otros productos como quínoa, calabaza, papa, zapallo, pallar, maní y algodón hacia los $400 \mathrm{AC}$ (Castro y Tarragó 1992).

Al norte de dicho cementerio, el asentamiento de Pircas está compuesto por conjuntos arquitectónicos funcionalmente diferenciados, donde los vegetales aparecen representados en capachos, cestos, fibras, cucharas, maíz, poroto, algarrobo y semillas de algodón, con fechas que fluctúan entre los 480 AC y los 500 DC (Núñez 1984; Castro y Tarragó 1992). Estas evidencias se suman a las obtenidas recientemente, consistentes en una notable presencia de artefactos de madera, incluyendo, entre otros, los mismos "colgantes" antropomorfos descritos anteriormente por Núñez (1984), junto a frutos de molle (Schinus molle), chañar (Geoffroea decorticans), tallos de brea (Tessaria absinthioides), calabaza (Lagenaria sp.), plantas acuáticas como cañaveral (Phragmites australis), qosqosa (Equisetum sp.) y junquillo (Scirpus sp.), además de semillas de herbáceas locales como oreja de chojchor (Exodeconus integrifolia), malva blanca (Tarasa operculata), yuyos (Familia Chenopodiaceae), pastos (Familia Poaceae) y las especies mencionadas por los autores anteriores (García et al. 2012).

Desde los restos vegetales así como desde otras líneas de evidencia, se puede observar cierta continuidad de la cultura material durante todo el primer milenio de nuestra era. En este sentido, consideramos que los contextos descritos constituyen el sustrato sobre el cual el Complejo Pica Tarapacá se erigió como sociedad y organizó su economía. Una situación diferente se expresaría luego, a partir del siglo XIII (fase Camiña, ca. 12501450 DC), evidenciado a partir de los asentamientos agrícolas reseñados al comienzo, de mayor densidad y emparentados con el Altiplano Meridional. Estos asentamientos corresponden a los poblados Nama, Camiña y Jamajuga, que son objetos de esta investigación, sumado a otros similares como Chusmiza, Mocha, Guaviña, Sibaya y Chiapa, entre otros, ubicados en la parte alta de la quebrada de Tarapacá (Niemeyer 1961; Núñez 1965, 1979; Moragas 1991, 1995; Uribe y Adán 2005; Adán et al. 2007).

\section{* Intervención arqueológica en los asentamientos Nama, CAmiña y Jamajuga}

Los recintos excavados de los cuales provienen los restos vegetales corresponden a los depósitos más abundantes y diversificados de los sitios trabajados. Asimismo, la mayor parte de ellos se ubica en los sectores más aglutinados, a saber el talud en el caso de Nama, el sector sur en Camiña y la ladera central en el caso de Jamajuga. Estos se vinculan constructivamente a categorías formales similares, generalmente de plantas ovaladas e irregulares con un tamaño del orden de los $20 \mathrm{~m}^{2}$ y muros de hilada simple; aunque hay algunos que alcanzan los $40 \mathrm{~m}^{2}$, así como otros que presentan muros con hilada doble y argamasa (Adán et al. 2007). Ninguno de ellos conserva techumbres, aunque, sobre la base de las formas tradicionales de construcción que aún se preservan en los valles y algunas evidencias arqueológicas al respecto, suponemos que debieron utilizarse vigas y postes de madera cubiertas de ramas, cañas o pajas. Una evidencia muy importante en este sentido, fue un poste hallado en el recinto 200 de Camiña (sector sur) que fue identificado como Aspidosperma desmanthum, ${ }^{3}$ un árbol de la vertiente oriental andina de madera color café anaranjada, reconocida por su peso y firmeza, que justifica su actual utilización en

\footnotetext{
3 La determinación taxonómica fue realizada por Mónica Rallo (Fac. Ciencias Forestales, Universidad de Chile).
} 
durmientes de ferrocarril y otras construcciones pesadas (García y Vidal 2006).

En primer lugar, el poblado arqueológico de Nama (UTM $\left.456232 \mathrm{E} ; 7878172 \mathrm{~N}^{4}\right)$ se emplaza sobre un cerro ubicado en la confluencia de las quebradas homónima y la quebrada Guayaquil (2.990 m.s.n.m.). Cuenta con 558 recintos organizados en dos sectores, el talud que concentra el $75 \%$ de los recintos y la cumbre, ambos circunscritos a un muro perimetral. El asentamiento se encuentra adyacente al pueblo actual, cuyos habitantes hacen uso de parte de las andenerías ubicadas a los pies del sitio, seguramente de data prehispánica. Asociadas a estas, se levantan cuatro torres funerarias o chullpas de adobe, de factura similar a las registradas en Sitani (Isluga) y en otros sectores altiplánicos sobre los 4.000 m.s.n.m. (Núñez 1965; Adán y Urbina 2006; Urbina y Adán 2006).

En este sitio se sondearon 12 recintos (pozos de 1x1 m adyacentes a los muros interiores), de los cuales nueve arrojaron material vegetal. En términos generales, los depósitos de Nama son delgados y poseen profundidades que oscilan entre los 4 y $71 \mathrm{~cm}$, con una media de $28 \mathrm{~cm}$, en cuya formación podría estar incidiendo la pronunciada pendiente del terreno. Por una parte, los recintos de la cumbre arrojaron depósitos más bien superficiales, con un promedio de $8 \mathrm{~cm}$ de profundidad y carente de fogones. Por otra parte, los recintos excavados en el talud presentaron depósitos diversos con distintas profundidades (hasta cinco estratos) y desigual comportamiento del material cultural. La estratigrafía en este sector evidencia en la mayoría de los casos un estrato inicial que corresponde al aterrazamiento artificial de la superficie del cerro, sobre el cual ocurre la ocupación; esta posteriormente es "sellada" luego del abandono del sitio por sedimentos de arrastre eólico y bloques de derrumbe de los muros (Méndez-Quirós y Uribe 2006).

En segundo lugar, Camiña (UTM 45416oE; 7864630N) se emplaza en el sector de Juanca, inmediatamente al oeste del pueblo homónimo actual, en la ladera norte de la quebrada que lleva el mismo nombre (2.412 m.s.n.m.). En este punto emana una vertiente que fue canalizada para el regadío de los andenes que se visualizan abando-

4 Todas las coordenadas se tomaron en Datum PSAD 56. nados en el lugar, los cuales debieron complementarse con los del fondo de valle, aún en uso por las comunidades actuales. El poblado cuenta con 588 recintos organizados en dos sectores, norte y sur, delimitados por una vía de circulación edificada. Se enfrenta hacia el este con el cerro Laymisiña, un hito significativo en el paisaje hasta la actualidad, donde se registraron seis chullpas de adobe, construidas de manera ascendente en la ladera y organizadas en pares. Asociado a estas, pero en el plano, se encuentra el cementerio del actual pueblo de Camiña superpuesto a un cementerio de fosas de piedras o cistas probablemente contemporáneo al poblado prehispánico (Niemeyer 1961; Núñez 1965; Adán y Urbina 2006; Urbina y Adán 2006).

En Camiña se sondearon 17 recintos (pozos de 1x1 m adyacentes a los muros interiores), todos los cuales arrojaron material vegetal. En términos generales, los depósitos presentaron profundidades que fluctúan entre los 12 y $83 \mathrm{~cm}$, con una media de $48 \mathrm{~cm}$. Estratigráficamente, los recintos ubicados en la cumbre y sector sur muestran que la ocupación principal está asociada a los estratos intermedios (p.e., recintos 139, 215, 238) o iniciales (p.e., 126); mientras que en los recintos del sector norte (p.e., recinto 296), esta se manifiesta superficialmente. Lo anterior podría estar indicando una historia ocupacional radial del asentamiento iniciado desde el sector más alto (Méndez-Quirós y Uribe 2006).

Por último, Jamajuga (UTM 479375E; 7780470N) se emplaza en la quebrada de Mamiña, aguas arriba del pueblo homónimo actual (2.850 m.s.n.m.). El poblado aprovecha uno de los cerros con mejores posibilidades visuales del entorno, captando incluso la cordillera de la Costa. Sin embargo, no alcanza la envergadura o monumentalidad de los asentamientos anteriormente descritos. Se contabilizaron un total de 135 recintos, los cuales se disponen siguiendo un patrón concéntrico en relación a la cumbre del cerro, la cual fue despejada para instalar una plaza (Niemeyer 1961; Urbina y Adán 2006). Al igual que en los otros casos, existe una asociación directa con las andenerías construidas en laderas y fondo de valle, que en este caso se encuentran completamente abandonadas. Aquí se sondearon cinco recintos (pozos de 1xı $\mathrm{m}$ adyacentes a los muros interiores), de los cuales únicamente la plaza (recinto $5 \mathrm{~A}$ ) y el recinto 76 arrojaron material vegetal. Los depósitos 
presentaron profundidades que fluctúan entre los 10 y $50 \mathrm{~cm}$, con una media $28 \mathrm{~cm}$, mostrando en general tres estratos depositados sobre el piso natural del cerro o aterrazamiento, a los que se asocia solamente una clara ocupación.

\section{* Criterios metodológicos para el TRATAMIENTO DEL MATERIAL VEGETAL}

En términos generales, el trabajo siguió en sus etapas de muestreo, identificación taxonómica y cuantificación, los métodos y técnicas delineadas por la escuela norteamericana (Dimbleby 1967; Hastorf y Popper 1988; Pearsall 1989). De esta manera, fueron analizados la totalidad de los macrorrestos vegetales recuperados tanto de harneros como por flotación. Esta última se realizó a base de un muestreo dirigido hacia los niveles estratigráficos con mayores evidencias orgánicas (rasgos), por una parte, y hacia los depósitos completos más representativos de la estratigrafía de cada sitio, por otra. Con ello se esperó tener una visión espacial y vertical de los contextos estudiados. Se flotaron un total de 125,5 litros de sedimentos, entre los cuales 62,8 provienen de Camiña, 47,0 de Nama y 15,7 de Jamajuga. Al mismo tiempo, del total flotado, 65,3 litros fueron obtenidos de los recintos muestreados por estrato (depósitos completos), mientras que los restantes 60,2 litros se obtuvieron de los recintos en los cuales solo fueron muestreados los niveles orgánicos o rasgos (Tablas 1, 2 y 3 ).

Los vegetales recuperados constituyen un universo medianamente heterogéneo compuesto por frutos, semillas, maderas y carbones. Solo los primeros dos grupos se lograron determinar taxonómicamente a partir de técnicas macroscópicas, gracias a que dichas evidencias han estado sometidas a procesos de deshidratación que les han permitido resguardar cicatrices que pueden ser imperecederas, posibles de ser observadas con lupa binocular $(0,7-4,5 \mathrm{X})$. En este mismo proceso, las muestras fueron comparándose con material de referencia actual colectado en las quebradas donde se emplazan los sitios arqueológicos y en la pampa del Tamarugal. En complemento, tuvimos acceso al herbario personal de Claudio Latorre (Facultad de Ciencias Biológicas, Pontificia Universidad Católica de Chile), quien además fue fundamental para guiar las identificaciones.
La comprensión de las evidencias recuperadas debe considerar, por una parte, una subrepresentación de los restos vegetales por efecto de las lluvias de verano y una sobrerrepresentación generada por efecto del viento. El primero afecta directamente la preservación de restos orgánicos mientras que el segundo lleva a que los depósitos incorporen semillas de herbáceas locales muy livianas y de fácil dispersión. Estas últimas tienden a distribuirse en los niveles superficiales, por lo cual las hemos considerado post-depositacionales. Tales especies corresponden a piyaya o cachiyuyo (Atriplex madariagae, Atriplex spp.), oreja de perro (Cistanthe amaranthoides), malva ( $T a-$ rasa operculata), lecheleche (Euphorbia sp.), tíkara hembra (Krameria lappacea), Malesherbia sp., Criptantha sp. y Sisymbrium sp.

Por último, el análisis intentó una mayor cercanía con lo local incluyendo antecedentes etnográficos recopilados por otros investigadores, cuya aplicación consideramos apropiada por cuanto propone un sustrato sobre el cual interpretar los datos más cercano que el nuestro y, sobre todo en el caso de las plantas, cuyo conocimiento se encuentra fuertemente arraigado a una cosmovisión que hunde sus raíces en el pasado prehispánico (van Kessel 1992; Castro y Varela 1994; Villagrán et al. 1999; Villagrán y Castro 2004).

\section{* Los vegetales en el contexto arqueológico}

Se determinaron un total de ocho especies vegetales correspondientes a plantas silvestres y cultivadas, todos ellas de origen local y posibles de obtener (recolectar o producir) al interior de las quebradas y en los pisos vegetacionales precordilleranos (2.600-4.000 m).

La muestra total se compone de 277 carporrestos, de los cuales 156 fueron registrados en Camiña (60\%), 58 en Nama (21\%) y 54 en Jamajuga (19\%). En relación a las especies silvestres, se registraron frutos y semillas tanto de algarrobo (Prosopis aff. chilensis) y q'ero (Escallonia angustifolia), como de herbáceas representadas por el malvisco (Cristaria dissecta). Entre los cultivos, se recuperaron mazorcas y granos de maíz (Zea mays), granos de quínoa (Chenopodium quinoa), una semilla de zapallo (Cucurbita sp.), cáscaras de calabaza (Lagenaria sp.) y fibra de algodón (Gossypium sp.). Todos ellos 
Contextos de uso de las plantas vinculadas al Complejo Pica Tarapacá, Andes Centro-Sur...

\begin{tabular}{|c|c|c|}
\hline Recinto & Nivel & Volumen (Litro) \\
\hline 67 & rasgo 1 & 2,1 \\
\hline 67 & rasgo 2 & 1 \\
\hline 183 & 1 & 1 \\
\hline 183 & 2 & 1 \\
\hline 183 & $3 \mathrm{~A}$ & 1 \\
\hline 183 & $3 \mathrm{~B}$ & 0,8 \\
\hline 183 & rasgo 1 & 0,8 \\
\hline 192 & rasgo 1 & 4,85 \\
\hline 251 & 1 & 1,2 \\
\hline 251 & 2 & 1 \\
\hline 251 & 3 & 1 \\
\hline 251 & 4 & 1 \\
\hline 251 & $2 \mathrm{~B}$ & 1 \\
\hline 251 & rasgo 1 & 5 \\
\hline 276 & rasgo 1 & 3,1 \\
\hline 276 & rasgo 2 & 0,3 \\
\hline 287 & rasgo 1 & 0,5 \\
\hline 287 & rasgo 2 & 1,1 \\
\hline 287 & rasgo 3 & 0,8 \\
\hline 287 & rasgo 4 & 9,5 \\
\hline 302 & rasgo 1 & 0,9 \\
\hline 363 & rasgo 1 & 7 \\
\hline $338 \mathrm{~A}$ & rasgo 1 & 1 \\
\hline TOTAL & & 46,95 \\
\hline
\end{tabular}

Tabla 1. Volumen de sedimentos flotados en el poblado de Nama.

\begin{tabular}{|c|c|c|}
\hline Recinto & Nivel & Volumen (Litro) \\
\hline $5 \mathrm{~A}$ & rasgo 1 & 6,2 \\
\hline 76 & 1 & 1 \\
\hline 76 & 2 & 1 \\
\hline 76 & $3 \mathrm{~A}$ & 1 \\
\hline 76 & $3 \mathrm{~B}$ & 1 \\
\hline 76 & 4 & 1 \\
\hline 76 & rasgo 1 & 4,5 \\
\hline TOTAL & & $\mathbf{1 5 , 7}$ \\
\hline
\end{tabular}

Tabla 2. Volumen de sedimentos flotados en el poblado de Jamajuga.

\begin{tabular}{|c|c|c|}
\hline Recinto & Nivel & Volumen (Litro) \\
\hline 126 & rasgo 1 & 1 \\
\hline 139 & Sup. & 1 \\
\hline 139 & 1 & 1,5 \\
\hline 139 & 2 & 1 \\
\hline 139 & 3 & 1,3 \\
\hline 139 & 4 & 1,4 \\
\hline 139 & $5 \mathrm{~A}$ & 1,3 \\
\hline 139 & ${ }_{5} \mathrm{~B}$ & 1 \\
\hline 139 & ${ }_{5} \mathrm{C}$ & 1,7 \\
\hline 139 & 6 & 1 \\
\hline 139 & $7 \mathrm{~A}$ & 1,4 \\
\hline 139 & $8 \mathrm{~B}$ & 1,4 \\
\hline 141 & rasgo I & 1 \\
\hline 200 & rasgo 1 & 5,95 \\
\hline 215 & rasgo 1 & 1,1 \\
\hline 215 & rasgo 2 & 1,3 \\
\hline 238 & Sup. & 1,1 \\
\hline 238 & 1 & 1,3 \\
\hline 238 & 2 & 1,3 \\
\hline 238 & $3 \mathrm{~A}$ & 1,1 \\
\hline 238 & $3 B$ & 1 \\
\hline 238 & rasgo 1 & 1 \\
\hline 238 & 4 & 1 \\
\hline 238 & 5 & 1,3 \\
\hline 238 & $6 \mathrm{~A}$ & 1,2 \\
\hline 238 & $6 \mathrm{~B}$ & 1,1 \\
\hline 238 & $7 \mathrm{~A}$ & 1,5 \\
\hline 238 & $7 \mathrm{~B}$ & 1,8 \\
\hline 238 & ${ }_{7} \mathrm{C}$ & 1 \\
\hline 238 & rasgo 2 & 1,9 \\
\hline 281 & rasgo 1 & 7,35 \\
\hline 296 & Sup. & 2,3 \\
\hline 296 & 1 & 2,1 \\
\hline 296 & $2 \mathrm{~A}$ & 1,9 \\
\hline 296 & $2 \mathrm{~B}$ & 1,5 \\
\hline 296 & $2 \mathrm{C}$ & 1,6 \\
\hline 308 & rasgo 1 & 1 \\
\hline 352 & rasgo 1 & 1,75 \\
\hline 381 & rasgo 1 & 1,4 \\
\hline TOTAL & & 62,85 \\
\hline
\end{tabular}

Tabla 3. Volumen de sedimentos flotados del poblado de Camiña. 
Magdalena García B., Mauricio Uribe R.

\begin{tabular}{|c|c|c|c|c|c|c|c|c|c|c|}
\hline \multirow{2}{*}{ Taxón } & \multirow{2}{*}{$\begin{array}{l}\text { Forma de } \\
\text { Crecimiento }\end{array}$} & \multirow{2}{*}{ Evidencia } & \multicolumn{2}{|c|}{ Nama } & \multicolumn{2}{|c|}{ Camiña } & \multicolumn{2}{|c|}{ Jamajuga } & \multicolumn{2}{|c|}{ Total } \\
\hline & & & $\mathbf{N}$ & $\%$ & $\mathbf{N}$ & $\%$ & $\mathbf{N}$ & $\%$ & $\mathbf{N}$ & $\%$ \\
\hline $\begin{array}{c}\text { ALGARROBO } \\
\text { Prosopis sp. }\end{array}$ & árbol & $\begin{array}{c}\text { vaina } \\
\text { (fragmento) }\end{array}$ & o & o & 4 & 100 & o & o & 4 & 100 \\
\hline $\begin{array}{l}\text { ALGARROBO } \\
\text { Prosopis sp. }\end{array}$ & árbol & semilla & 2 & 3,07 & 63 & 96,92 & o & o & 65 & 100 \\
\hline $\begin{array}{l}\text { Q'ERO Escallonia } \\
\text { angustifolia }\end{array}$ & árbol & fruto & 11 & 16,17 & 57 & 83,82 & o & ० & 68 & 100 \\
\hline $\begin{array}{l}\text { MALVISCO } \\
\text { Cristaria dissecta }\end{array}$ & hierba & semilla & 5 & 29,41 & 5 & 29,41 & 7 & 41,17 & 17 & 100 \\
\hline $\begin{array}{l}\text { MAÍZ } \\
\text { Zea mays }\end{array}$ & cultivo & mazorca & 3 & 15,78 & 15 & 78,94 & 1 & 5,26 & 19 & 100 \\
\hline $\begin{array}{l}\text { MAÍZ } \\
\text { Zea mays }\end{array}$ & cultivo & grano & 13 & 32,5 & 19 & 47,5 & 8 & 21 & 40 & 100 \\
\hline $\begin{array}{l}\text { QUÍNOA } \\
\text { Chenopodium } \\
\text { quinoa }\end{array}$ & cultivo & semilla & 26 & 30,23 & 21 & 24,41 & 39 & 45,34 & 86 & 100 \\
\hline $\begin{array}{l}\text { ZAPALLO } \\
\text { Cucurbitasp. }\end{array}$ & cultivo & semilla & 1 & 100 & o & o & o & ० & 1 & 100 \\
\hline $\begin{array}{l}\text { ALGODÓN } \\
\text { Gossypium sp. }\end{array}$ & cultivo & $\begin{array}{c}\text { fibra } \\
\text { (fragmento) }\end{array}$ & o & 0 & 1 & 100 & o & o & 1 & 100 \\
\hline $\begin{array}{l}\text { CALABAZA } \\
\text { Lagenaria sp. }\end{array}$ & cultivo & $\begin{array}{c}\text { cáscara } \\
\text { (fragmento) }\end{array}$ & o & o & 30 & 100 & o & o & 28 & 100 \\
\hline
\end{tabular}

Tabla 4. Diversidad de especies botánicas identificadas y su presencia relativa en cada asentamiento.

están presentes en los sedimentos de Camiña, excepto el zapallo que se encuentra únicamente en Nama. Junto a este, Nama también arrojó restos de malvisco, maíz y quínoa, que fueron también identificados en Jamajuga (Tabla 4).

Con relación a la presencia relativa de cada especie en los distintos asentamientos, podemos señalar que el 97\% del algarrobo (Prosopis sp.) se distribuye en Camiña y solo el 3\% lo hace en Nama. Una tendencia similar reproducen los frutos de q'ero (Escallonia angustifolia) al distribuirse el $84 \%$ en Camiña y $16 \%$ en Nama. El malvisco (Cristaria dissecta), por su parte, está presente en todos los contextos estudiados, entre los cuales el 59\% se distribuye en similar proporciones en Nama y Camiña y un $41 \%$ en Jamajuga.

En relación a los cultivos, los granos de maíz (Zea mays) están representados en todos los asentamientos, concentrándose un $45 \%$ en Camiña, un 34\% en Nama y un 21\% en Jamajuga. En cuanto a los granos de quínoa (Chenopo- dium quinoa), un $45 \%$ se asocia a Jamajuga (exclusivamente en la plaza), un $30 \%$ a Nama y un $24 \%$ a los recintos de Camiña.

El análisis de densidad se elaboró a partir de los carporrestos recuperados por flotación, del cual se marginaron los fragmentos de vaina de algarrobo (Prosopis sp.), pericarpios de calabaza (Lagenaria sp.) y fibra de algodón (Gossypium sp.), por no constituir unidades cuantificables. De este modo, la mayor densidad de restos se halló en la plaza de Jamajuga con un índice de 3,4 carporrestos por litro flotado (carp/lt), seguido por Camiña con 2,6 carp/lt y finalmente Nama con 1,2 carp/lt. Si bien bajos, los valores son coherentes con la mayor participación que habría en las actividades desarrolladas en la plaza de Jamajuga.

Respecto a los usos etnográficos, de un total de ocho plantas identificadas, cinco de ellas poseen un valor alimenticio (algarrobo, maíz, quínoa, zapallo y calabaza). Le sigue en importancia, representado por tres especies cada uno, los valores artesanal (algarrobo, zapallo y calabaza) 


\begin{tabular}{|c|c|c|c|c|c|c|c|c|c|}
\hline & ART & $\mathrm{COM}$ & $\mathrm{CON}$ & FOR & LEÑ & MED & RIT & TEX & TOTAL \\
\hline $\begin{array}{c}\text { ALGARROBO } \\
\text { Prosopissp. }\end{array}$ & & $\mathrm{X}$ & $\mathrm{X}$ & $\mathrm{X}$ & $\mathrm{X}$ & $\mathrm{X}$ & & & 5 \\
\hline $\begin{array}{l}\text { Q'ERO } \\
\text { Escallonia angustifolia }\end{array}$ & & & $\mathrm{X}$ & & $X$ & & & & 2 \\
\hline $\begin{array}{l}\text { MALVISCO } \\
\text { Cristaria dissecta }\end{array}$ & & & & $\mathrm{X}$ & & & & & 1 \\
\hline $\begin{array}{l}\text { MAÍZ } \\
\text { Zea mays }\end{array}$ & & $X$ & & $\mathrm{X}$ & X & $\mathrm{X}$ & $\mathrm{X}$ & & 5 \\
\hline $\begin{array}{c}\text { QUÍNOA } \\
\text { Chenopodium quinoa }\end{array}$ & & $X$ & & $\mathrm{X}$ & $\mathrm{X}$ & $\mathrm{X}$ & $\mathrm{X}$ & & 5 \\
\hline $\begin{array}{l}\text { ZAPALLO } \\
\text { Cucurbita sp. }\end{array}$ & $X$ & $X$ & & & & & & & 2 \\
\hline $\begin{array}{l}\text { ALGODÓN } \\
\text { Gossypium sp. }\end{array}$ & & & & & & & & $\mathrm{X}$ & 1 \\
\hline $\begin{array}{l}\text { CALABAZA } \\
\text { Lagenaria sp. }\end{array}$ & $\mathrm{X}$ & X & & & & & & & 2 \\
\hline
\end{tabular}

Tabla 5. Claves de los usos etnográficos asignados a las hierbas, árboles y cultivos identificados en los contextos arqueológicos. ART: artesanal; COM: comestible; CON: construcción; FOR: forrajero; LEÑ: leña; MED: medicinal; RIT: ritual; TEX: textil (Villagrán et al. 1999; Villagrán y Castro 2004).

y forrajero (malvisco, maíz y quínoa); luego, representado por dos especies, se encuentran los usos constructivos y combustibles (algarrobo y q'ero) y el valor ceremonial (maíz y quínoa). Y, finalmente, el uso textil representado únicamente por el algodón (Tabla 5)

\section{Nama}

En Nama, los vegetales se distribuyen principalmente en los recintos 67 y 251 . Con respecto al primero, las evidencias provienen del rasgo 1 (fogón), concentrando la mayor densidad de carporrestos del sitio equivalente a 9 carp/lt. A este contexto se asocian fragmentos de mazorca y doce granos de maíz (92\%), junto a 16 granos carbonizados de quínoa $(62 \%)$ y alimentos de origen animal como camélido no identificado, chinchilla (Chinchilla sp.) y vizcacha (Lagidium viscacia), equivalentes a un individuo cada uno (González 2006). El fogón fue fechado en cal. 1160-1310 DC (Méndez-Quirós y Uribe 2006).

Por otra parte, en el recinto 251, donde se aplicó el muestreo por estrato, el depósito total dio un índice de densidad de 1,5 carp/lt, superior al promedio general del sitio. De este contexto se recuperaron dos semillas de algarrobo (100\%), diez frutos de q'ero (91\%), dos semillas carboni- zadas de malvisco (40\%), restos de mazorca de maíz, un grano carbonizado de quínoa (4\%) y una semilla de zapallo (100\%), junto con fragmentos de cerámica Pica Charcollo, Pica Gris Alisado, Isluga Rojo Revestida y Pocoma, desechos líticos, restos óseos, malacólogicos y pigmento (Figuras 2, 3 y 4). En torno al comportamiento estratigráfico de los materiales, estos tienden a concentrarse en los niveles intermedios, entre las capas 2 y 3 . Por su parte, el nivel 4 o inicial de la ocupación no arrojó ninguna clase de evidencia, mientras que los niveles superficiales se relacionan únicamente a elementos vegetales post-depositacionales (Atriplex sp., Malesherbia sp., Tarasa operculata).

\section{Camiña}

En Camiña los recintos que concentran la mayor abundancia de material vegetal son las estructuras 126, 215, 139 y 238 . En el recinto 126 , los restos se asocian al rasgo I (fogón) donde se ha recuperado la mayor densidad de carporrestos del sitio (17 carp/lt). A este contexto se asocia un fruto de q'ero (2\%), cuatro semillas de malvisco (80\%), dos mazorcas (13\%) y dos granos de maíz (11\%), diez granos carbonizados de quínoa (48\%) y nueve fragmentos de calabaza (30\%), además de restos cerámicos, óseos, líticos y malacológicos. 


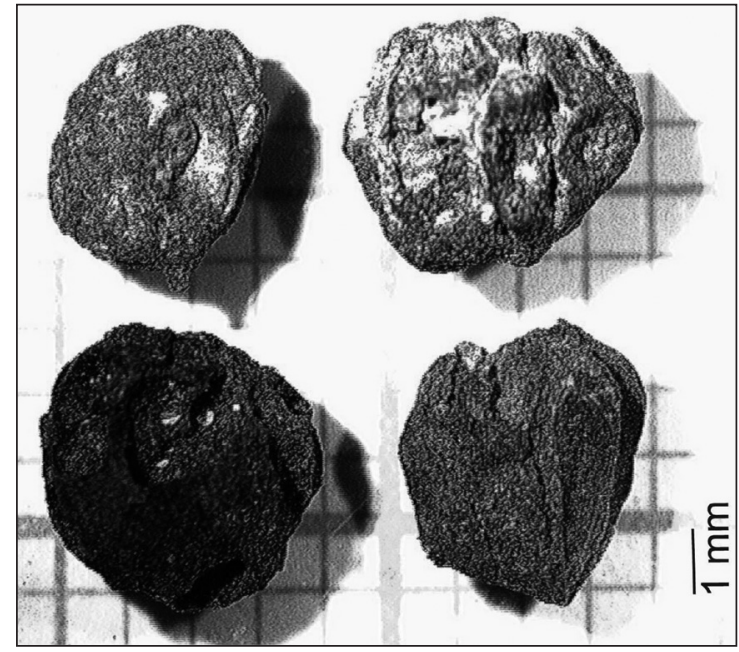

Figura 2. Frutos de q'ero (Escallonia angustifolia). Nama, recinto 251 nivel $2 \mathrm{~B}$.

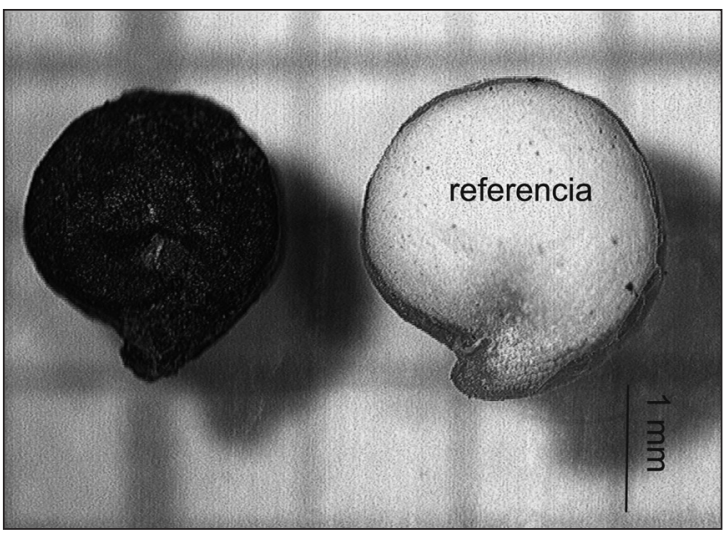

Figura 3. Granos carbonizados de quínoa (Chenopodium quinoa). Nama, recinto 251 nivel 2B.

En el recinto 215, los restos provienen del interior de dos grandes vasijas halladas enterradas in situ. En promedio, ambas arrojan una densidad de 8,3 carp/lt. De ellas se extrajeron seis semillas de algarrobo (10\%), siete frutos de q'ero (12\%), una mazorca completa (7\%), junto a cinco granos de maíz (26\%), dos quínoas (10\%) y 16 fragmentos de calabaza (53\%). Asociado a esta diversidad, en una de las vasijas se halló un rico contexto compuesto por preformas de cuentas confeccionadas en Oliva peruviana junto a desechos de talla lítica y perforadores de sílice (Valenzuela 2009; Carrasco 2005 MS).

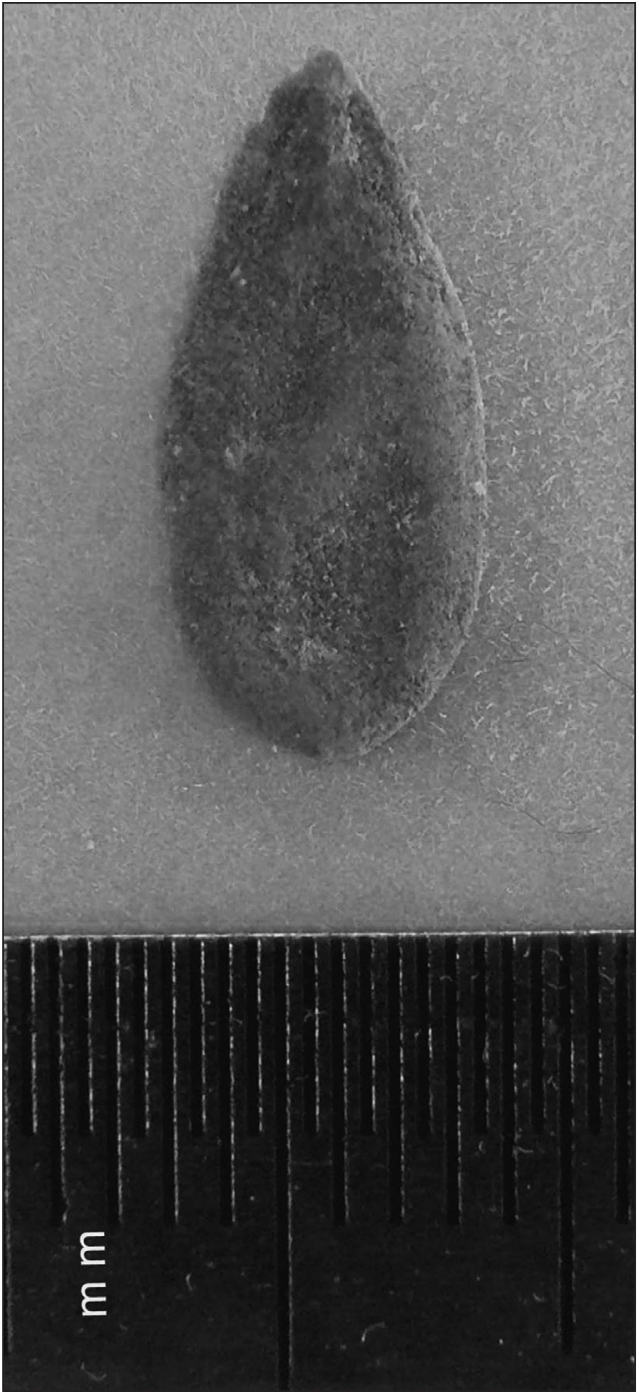

Figura 4. Semilla de zapallo (Cucurbita sp.). Nama, recinto 251 nivel 3.

En los recintos 139, 238 y 296 se aplicó el muestreo por estrato dando un índice de densidad de 4,8 carp/lt, 1,64 carp/lt y o carp/lt, respectivamente. En términos generales, al depósito del recinto 139 se asocian once semillas de algarrobo (71\%), trece frutos de q'ero (19\%), una semilla de malvisco (20\%), once mazorcas de maíz (73\%) y ocho granos de esta misma especie (42\%), tres granos de quínoa (14\%) y tres fragmentos de calabaza (7\%) (Figura 5). Estos se asocian principalmente a los niveles intermedios, entre las capas 3 y 6 , donde también se hallaron alimentos de origen animal como camélido no identifi- 


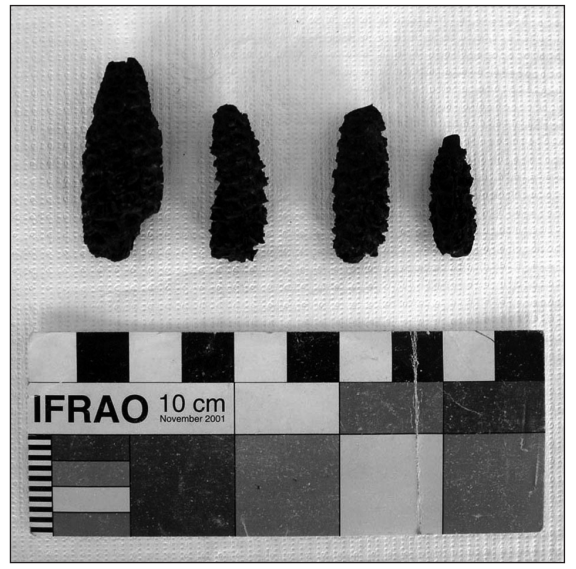

Figura 5. Mazorcas carbonizadas de maíz (Zea mays). Camiña, recinto 139 nivel $5 \mathrm{~A}$.

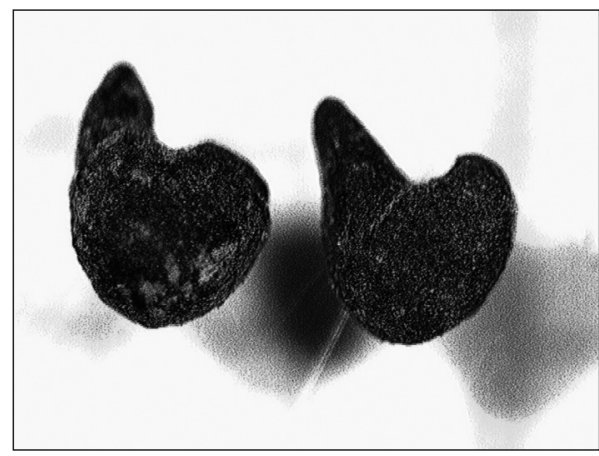

Figura 6. Semillas carbonizadas de malvisco (Cristaria dissecta). Plaza de Jamajuga, rasgo 1.

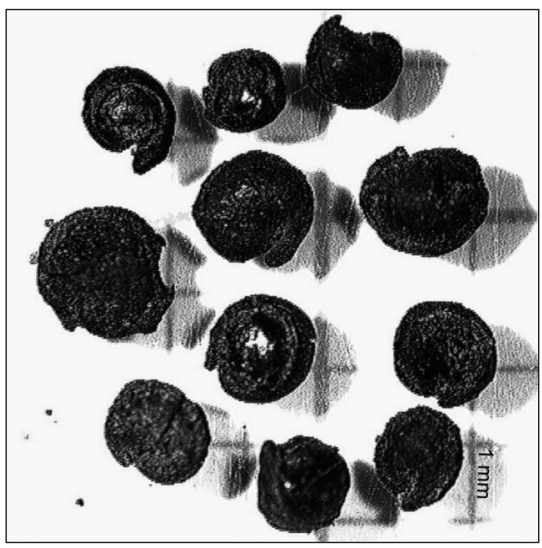

Figura 7. Granos carbonizados de quínoa (Chenopodium quinoa). Plaza de Jamajuga, rasgo 1. cado y chinchilla (Chinchilla sp.) (González 2006), un fragmento de mineral de cobre, desechos de talla lítica y fragmentos de cerámica doméstica local (Pica Charcollo, Isluga Sin Decoración), así como de Atacama (Dupont) y Arica (Arica No Decorado, Pocoma Gentilar), (Uribe et al. 2007). La capa 3-4 fue fechada en cal. 1200-1400 DC (Méndez-Quirós y Uribe 2006).

De acuerdo a lo anterior, estratigráficamente la única evidencia presente durante toda la ocupación es el maíz, que se encuentra presente desde la capa 8 hasta la capa 1, esta última únicamente asociada a especies post-depositacionales dadas indistintamente por Atriplex sp., Cistanthe amaranthoides, Criptantha sp., Malesherbia sp. y Tarasa operculata.

Al recinto 238, el depósito más profundo del sitio $(83 \mathrm{~cm})$, se asocian nueve semillas de algarrobo (14\%), 16 frutos de q'ero $(28 \%)$, tres granos de maíz $(16 \%)$ y fragmentos de mazorca, un grano de quínoa (5\%), tres fragmentos de calabaza (10\%) y fibra de algodón (100\%). Asociado a ellos, el depósito arrojó restos cerámicos, líticos, óseos, malacológicos, guano, textil y pigmento. En términos estratigráficos, los restos vuelven a concentrarse en los niveles intermedios, entre las capa 3 y 6 , donde se encuentran representadas todas las especies mencionadas. En este depósito no se hallaron evidencias en los niveles superficiales, solo especies post-depositacionales, mientras que en el inferior o inicial, únicamente un fruto de q'ero (capa $7 \mathrm{C}$ ).

\section{Jamajuga}

En Jamajuga, los vegetales se asocian al recinto 76 y a la plaza ubicada en la cumbre del poblado (Estructura $5 \mathrm{~A}$ ). Al primero se le aplicó el muestreo por estrato que resultó en una densidad de 3,4 carp/lt, referido únicamente a tres semillas carbonizadas de malvisco (43\%). No se hallaron fogones (rasgos) en ninguno de los recintos menores excavados.

En la plaza, en cambio, se registró la mayor densidad de restos asociado al rasgo 1 (7,4 carp/lt), donde se encontraron cuatro semillas de malvisco (57\%), tres granos de maíz (37,5\%), fragmentos de mazorca (100\%) y 39 granos de quínoa (100\%), todos ellos completamente carbonizados (Figuras 6 y 7). Se suman además cinco granos de maíz $(62,5 \%)$ asociados a la capa 1 y 3 y huesos calcinados de camélido no identificado. Destaca la total ausencia de 
otros alimentos de uso doméstico como algarrobo, calabaza y chinchilla, entre otros, asociados a los recintos menores de los sitios anteriores. Junto con ello, se suma la recurrencia de fragmentos de cerámica local y foránea de uso doméstico y ceremonial, incluyendo tipos Chilpe, San Miguel y Pocoma (Uribe et al. 2007). Por último, resultan sugerentes el conjunto de cistas construidas en el interior de la plaza, las cuales en sitios contemporáneos de la sierra de Arica han sido interpretadas en relación al almacenaje comunal (Muñoz y Chacama 2006). El contexto se asocia a una estratigrafía compuesta por capas sucesivas de ceniza y carbón que se extienden por toda la unidad de excavación (1x1 m), el cual fue fechado en cal. 1160-1290 DC (Méndez-Quirós y Uribe 2006).

\section{* Discusión}

Las especies identificadas señalan que durante el período Intermedio Tardío los habitantes de los sitios Nama, Camiña y Jamajuga llevaron a cabo actividades agrícolas y de recolección como parte de sus prácticas cotidianas. Por una parte, la recolección de especies silvestres se mantendría vigente y se orientaría principalmente a especies arbóreas de las quebradas, como q'ero (Escallonia angustifolia) y algarrobo (Prosopis sp.). Estas especies, cuyos restos fueron hallados en Nama y Camiña, debieron ser demandados por la dureza de sus maderas tanto para construcción como para combustible, y por la relevancia alimenticia de las vainas de este última. Por otra parte, el malvisco (Cristaria dissecta) está presente en bajas proporciones en Nama, Camiña y Jamajuga a partir de semillas carbonizadas que evocan su utilización como combustible y forraje. De todos modos, si bien la recolección es un ámbito de escasa visibilidad arqueológica en comparación con la agricultura, no podemos desconocer su importancia en términos de la amplitud de contextos en que estas son requeridas como alimento, medicina, forraje, combustible y como materia prima (Villagrán y Castro 2004).

En un ámbito complementario, la agricultura es cuantitativa y cualitativamente más visible. En términos generales, el cultivo más recurrente es el maíz (Zea mays) que está presente en todos los asentamientos trabajados, donde un $47 \%$ de los granos cuantificados se hallaron en Camiña, un $32 \%$ en Nama y un 20\% Jamajuga. Le siguen en importancia los restos de quínoa (Chenopodium quinoa) distribuidos en Jamajuga, Nama y Camiña, donde esta alcanza, $45 \%, 30 \%$ y $24 \%$, respectivamente. Se suman ciertas evidencias únicas como los 28 fragmentos de cáscara de calabaza (Lagenaria sp.) y un pequeño fragmento de fibra de algodón (Gossypium sp.), ambos en Camiña, y una semilla de zapallo (Cucurbita sp.) en Nama.

En cuanto al comportamiento de los restos, destaca en primer lugar, la preeminencia de maíz y quínoa en relación a los demás productos cultivados que, en general, muestran frecuencias poco significativas. Lo anterior establece una diferencia importante con respecto al comportamiento de los cultivos asociados al poblado Caserones (fase Tarapacá, ca. 950-1250 DC), donde las distintas especies allí representadas poseen una distribución homogénea. En este sentido, creemos que en los sitios estudiados, los cultivos tales como zapallo, calabaza o algodón, entre otros, pudieron producirse a baja escala, eventualmente orientada al autoconsumo o, de otro modo, pudieron obtenerse desde los valles bajos donde se documentan asentamientos contemporáneos como Tarapacá Viejo (Adán et al. 2007; Vidal 2008).

Ahora, en términos más amplios, los contextos destacados en los tres poblados estudiados arrojaron una asociación recurrente de maíz, quínoa, camélido, cerámica Pica Charcollo y carbón. Al patrón anterior, se irán sumando otras materialidades que permiten reconocer dos contextos de uso asociados al maíz y la quínoa. En primer lugar, relevamos un tipo de contexto que consideramos doméstico o familiar, de preparación y consumo de alimentos asociado generalmente a fogones situados en los márgenes interiores de las viviendas, los cuales arrojan además restos de otros alimentos (p.e., algarrobo, zapallo, calabaza, camélido, vizcacha y chinchilla), junto a fragmentos de cerámica doméstica de origen local (Pica Charcollo, Pica Gris Alisado) y altiplánica (Isluga Sin Decoración), lascas primarias y desechos de talla bifacial.

Un segundo contexto de uso se asociaría a una dimensión que consideramos pública o comunitaria, como se expresa en la plaza de Jamajuga, donde el maíz y la quínoa aparecen asociados a extensas quemas realizadas adyacentes al área de cistas interpretadas como parte del almacenaje comunal. Junto a ellos, se asocian además restos de camélido calcinado y fragmentos de cerámica local y foránea de uso doméstico (tipos Pica Charcollo e Isluga 
Sin Decoración) y ceremonial (tipo Chilpe, San Miguel y Pocoma Gentilar), que en gran medida corresponden a desechos de botellas, jarros y cántaros. Este contexto podría vincularse a eventos comunales de redistribución, donde los alimentos mencionados podrían estar siendo requeridos en términos de su eficacia simbólica, del mismo modo como la chicha de maíz que eventualmente estaría contenida en esta clase de vasijas.

Como insinuamos anteriormente, ambos contextos de uso evocan un ordenamiento material diferente al registrado en las aldeas de épocas previas. Frente a una agricultura extensiva en productos semitropicales diversos, las presentes evidencias sugieren una dinámica agrícola mayormente especializada en torno a los productos destacados como maíz y quínoa. Al mismo tiempo, tanto la producción, distribución así como el consumo de estos productos debió organizarse de manera menos circunscrita que en tiempos previos, ya que aquí la presencia de elementos foráneos, del Altiplano, Arica e incluso Atacama, adquieren un claro protagonismo.

\section{$*$ Conclusiones}

Los restos vegetales analizados dan cuenta que ante todo se hizo uso de los recursos vegetales disponibles en el área circundante a los asentamientos y, en este sentido, consideramos que la mayor parte de las especies identificadas son locales; es decir, obtenidas del interior de las quebradas y cerros aledaños, que desde la percepción del paisaje que poseen las comunidades andinas corresponden fundamentalmente a las etnocategorías de campo y chakra (Villagrán y Castro 2004).

En relación con la distribución de los restos, señalamos que en Nama, la distribución de los vegetales se concentra en el sector oeste del talud, donde se encuentran representadas todas las especies identificadas para el asentamiento. Los recintos que alcanzan mayor diversidad son el 67 (rasgo 1) y 251 (depósito completo). El primero concentra la mayor densidad de carporrestos de la muestra total, con un índice de 9 carp/lt. Asimismo, ambos reúnen la mayor parte de las evidencias de maíz (Zea mays), quínoa (Chenopodium quinoa), zapallo (Cucurbita sp.), q'ero (Escallonia angustifolia), algarrobo (Prosopis sp.) y malvisco (Cristaria dissecta) del asentamiento.
En Camiña los restos se concentran en el sector sur, donde están presentes todas las especies representadas del asentamiento. De ese sector, destacamos los depósitos excavados en los recintos 126 (rasgo 1), 139, 215 (rasgo 1 y 2) y 238 probablemente correspondientes a viviendas y/o espacios multifuncionales, donde hay una asociación recurrente de maíz (Zea mays), quínoa (Chenopodium quinoa), algarrobo (Prosopis sp.), q'ero (Escallonia angustifolia) y malvisco (Cristaria dissecta). Estratigráficamente, los restos tienden a agruparse en los niveles intermedios y, en la mayoría de los casos, se asocian a basuras primarias que implican un consumo in situ. La mayor densidad de carporrestos la encontramos al interior de las vasijas del recinto 215 , las cuales arrojaron un índice de 8,3 carp/lt, constituyéndose como el segundo contexto más denso de la muestra.

Por último, en Jamajuga los restos vegetales provienen principalmente del rasgo 1 (quemas) de la plaza, que arrojó un índice de densidad de 7,4 carp/lt. Se asocian a este un conjunto de granos de maíz (Zea mays) y quínoa (Chenopodium quinoa) junto con semillas carbonizadas de malvisco (Cristaria dissecta), entre otros.

Si bien las frecuencias son bajas debido a los problemas de preservación, las dos estrategias de muestreo adoptadas logran resultados coherentes, ya que la mayor densidad de carporrestos se asocia a los fogones y contenedores de almacenaje (rasgos), los cuales alcanzan un promedio de 3,5 carp/lt, con extremos entre o a 9 carp/lt. Por su parte, los recintos escogidos para muestrear la totalidad de sus depósitos arrojaron un promedio de 2 carp/lt, con extremos entre o y 4,8 carp/lt. De este modo, todos los taxones identificados se asocian indistintamente a ambos elementos estratigráficos, capas y rasgos, pero su distribución estratigráfica permite reconocer ocupaciones con distintos grados de intensidad. La tendencia general de todos ellos es concentrarse en los niveles estratigráficos intermedios (entre las capas 3 y 4), con niveles iniciales poco significativos en términos vegetales (entre capas 8 y 5 según los contextos), y niveles superficiales dados por derrumbes de muros y semillas livianas que debieron ingresar post-depositacionalmente por efecto del viento.

Lo anterior nos permite retomar y discutir ciertas apreciaciones acerca de la época de nuestro interés. El Norte Grande en el Intermedio Tardío ha sido tradicionalmente vinculado con los procesos de los Andes Centrales, y en 
especial a la situación de la subárea Circun-Titicaca. Se asume, por lo tanto, un traslape de influencias Tiawanaku sobre desarrollos locales y la incorporación al territorio de nuevos grupos altiplánicos, las que se definen como situaciones diferenciales, permanentes o transitorias. Es una dinámica regida por el entrecruzamiento de varias esferas de interacción orientadas por el patrón generalizado de complementariedad de recursos (Schiappacasse et al. 1989). La anterior concepción, constituida en una suerte de paradigma de las sociedades andinas preincaicas, empleó como armazón teórico el auge experimentado por los estudios etnohistóricos andinos, especialmente a partir de las contribuciones de Murra y sus estudios sobre las sociedades agropecuarias del altiplano. Sin embargo, nuestra lectura observa que lo que en un principio se constituyó en un fundamental estímulo para la arqueología de las sociedades andinas y el ejercicio interdisciplinario, se expresa en la actualidad en la carencia de interpretaciones sobre los sistemas sociales del Intermedio Tardío a partir de la cultura material propiamente tal (Uribe 2006).

En este caso específico, se ha planteado tradicionalmente que las comunidades agrícolas de las quebradas y oasis interiores de Tarapacá, a partir de su excedente, habrían integrado un sistema de complementariedad con los asentamientos de la sierra y el altiplano, lo que habría permitido el aprovechamiento racional y la circulación de los recursos de los diferentes ambientes. Así, en este territorio se habrían generado puntos terminales obligados para el acceso a importantes recursos y su intercambio, cuya relevancia quedaría plasmada en geoglifos y petroglifos especialmente en el interior $y$, con ello, una notable concentración de rutas de caravanas de llamas a lo largo de la pampa. Este sistema se habría caracterizado consecuentemente por una "armonía" en términos de la circulación de recursos, la integración económica, la redistribución y el desarrollo social, político y cultural, desencadenando la complejidad social, organizados y jerarquizados en "señoríos" (Núñez y Dillehay 1995: 147) de pequeña y mediana escala, económicamente interdependientes.

Las debilidades de este modelo han sido expuestas por sus mismos autores, quienes señalan, por ejemplo, que la Movilidad Giratoria “...tiene limitaciones obvias. Se concentra, casi exclusivamente, en aspectos económicos y demográficos del área de estudio, proporcionando escasa información sobre organización política o social. Existen razones para esto. Se ha trabajado tan poco en áreas de actividad intra e intersitios, patrones de asentamiento y de residencia, y bienes de estatus de grupos de élite y noélite, que solo es posible especular acerca del tipo y nivel de la organización social y política que existió..." (Núñez y Dillehay 1995: 150). Los enfoques mencionados privilegian aspectos económicos y ecológicos, lo cual solo representa parte de esta realidad cultural. Asimismo, agrega Albarracín-Jordán, "Las explicaciones acerca de los cambios en el asentamiento humano a través del tiempo no pueden centrarse únicamente en la relación cultura-medio ambiente, descuidando las relaciones sociales como elementos dinámicos y de cambio" (1996: 81).

Es necesario, entonces, un acercamiento a las fuerzas sociales que producen la cultura material y cómo recíprocamente esta contribuye a su estructura y procesos sociales para acceder a un conocimiento más profundo y asertivo acerca del papel de la materialidad en los procesos de formación de las identidades sociales y étnicas, como su expresión y reproducción en el pasado. Frente a este panorama, la información aquí proporcionada por la muestra arqueobotánica nos permite elaborar una opinión que se desprende exactamente del contexto material y que aporta a la comprensión arqueológica del Complejo Pica Tarapacá del Intermedio Tardío. Por una parte, se confirma que existe una producción generalizada de ciertas plantas cultivadas, específicamente del maíz y la quínoa; las que aparecen representadas en diversos contextos sociales, tanto domésticos y familiares, así como públicos y comunitarios, en asentamientos de distinta envergadura. Por lo tanto, la importancia de estas plantas es innegable en el ámbito social. No obstante, la densidad de su consumo impide sustentar por el momento que su producción estaba asociada a una escala excedentaria $y$, más aún, que esta sostenía un sistema de intercambio institucional que articulaba los pisos ecológicos y sus recursos del altiplano hasta la costa.

Con lo anterior, no estamos negando que la especialización agrícola y las prácticas caravaneras e intercambio existieran, sino que dichas actividades pudieron tener un carácter distinto o estar bajo otras prácticas de la esfera social más significativas y explícitas a nivel de asentamiento. De lo contrario, habría sido esperable encontrar grandes concentraciones del material arqueobotánico en contextos de almacenaje y/o insinuando lugares de prepa- 
ración de los mismos y no solo de consumo. Como consecuencia de ello, creemos que los asentamientos estudiados refieren a prácticas más sociales que económicas, destinadas a la reproducción social de sus unidades domésticas y de la comunidad misma, correspondiente a la dinámica segmentaria que mejor caracterizada a las sociedades del Intermedio Tardío (Albarracín-Jordán 1996). Desde esta perspectiva, los colectivos no estaban empecinados solo en la tarea productiva, sino que se constituían en su permanente relación de contrariedad e igualdad entre las familias y la comunidad, estampada en cada uno de sus poblados y maneras de consumir lo producido. Sin duda, para avanzar y validar esta propuesta ahora, se requiere profundizar en la dimensión simbólica de estas plantas, un recorrido bastante adelantado por la etnología andina pero una deuda todavía pendiente de la arqueología.

Agradecimientos A Victoria Castro por guiar esta investigación. A Alejandra Vidal por el apoyo en el análisis de los restos vegetales. A Claudio Latorre por su contribución en la determinación taxonómica y por brindar bibliografía especializada para ello. A Rolando Ajata por la elaboración de la Figura 1. Finalmente, a los evaluadores anónimos que contribuyeron a mejorar notablemente este artículo. La investigación fue financiada por los proyectos FONDECYT 1030923 y 1080458.

\section{$*$ Referencias citadas}

ACUTO, F., 2007 Fragmentación vs. Integración comunal: repensando el período Tardío del Noroeste Argentino. Estudios Atacameños 34: 71-95.

ADÁN, L., y S. URBINA, 2006. Arquitectura quebradeña del Complejo Pica-Tarapacá. Modos de hacer, opciones de diseño, rasgos significativos y decisiones funcionales. Ponencia presentada en el XVII Congreso de Arqueología Chilena, Valdivia.

ADÁN, L., S. URBINA y M. URIBE, 2007. Arquitectura pública y doméstica en las quebradas de Tarapacá, asentamiento y dinamismo social en el Norte Grande de Chile. En Taller de Procesos Sociales Prehispánicos en los Andes Meridionales, A. Nielsen, M. C. Rivolta, P. Mercolli, M. Vázquez y V. Seldes (Eds.). Editorial Bayas, Córdova, Argentina.

ALBECK, M. E., 2011. Estudios de agricultura prehispánica en Casabindo (1980-1993). En Arqueología de la Agricultura. Casos de estudio en la región andina argentina, M. A. Korstanje y M. Quesada (eds.), pp. 12-47. Ediciones Magna, Santiago.

BRINER, C., 1985. Caracterización fenotípica de los biotipos de Tamarugo en la Pampa del Tamarugal. En Estado actual del conocimiento sobre Prosopis tamarugo. Corporación Nacional Forestal, Universidad de Tarapacá, Arica.

CARRASCO, C., 2005 Ms. Materialidad lítica de sitios tardíos de la región cultural de Tarapacá, norte de Chile. Informe proyecto FONDECYT 1030923. Manuscrito en poder del autor.

CASTRO, V., 2002. Ayquina y Toconce: paisajes culturales del norte árido de Chile. En Paisajes Culturales en los Andes, pp. 209-222. Representación de la UNESCO en Lima, Perú.
CASTRO, V., y M. TARRAGÓ, 1992. Los inicios de la producción de alimentos en el Cono Sur de América. Revista de Arqueología Americana 6: 91-124.

CASTRO, V., y V. VARELA, 1994. Ceremonias de tierra y agua. Ritos milenarios andinos. Fondart/ Fundación Andes, Kuppenheim, Santiago.

DimblebY, G., 1967. Plants and Archaeology. The Archaeology of the Soil. Paladin, London.

GARCÍA, M., y A. VIDAL, 2006. Distribución y contextos de uso de las plantas arqueológicas del asentamiento Camiña-1 (12501450 DC), región de Tarapacá. Actas del XVII Congreso Nacional de Arqueología Chilena. Ediciones Kultrún, Valdivia.

GARCÍA, M., A. VIDAL, A. MALDONADO, M. P. PEÑA y V. MANDAKOVIC. Economías domésticas y tecnologías vegetales en las aldeas de la pampa del Tamarugal: dos expresiones formativas para Tarapacá (ca. 200 AC-900 DC). Enviado a Estudios Atacameños.

GONZÁLEZ, J., 2006. Arqueofauna del período Intermedio Tardío: Complejo Pica Tarapacá. Actas del XVII Congreso Nacional de Arqueología Chilena. Ediciones Kultrún, Valdivia.

HASTORF, C., y V. POPPER, 1988. Current Paleoethnobotany. Analytical Methods and Cultural Interpretations of Archaeological Plant Remains. The University of Chicago Press, Chicago.

MÉNDEZ-QUIRÓS, P., y M. URIBE, 2006. Análisis estratigráfico y cronología del complejo cultural Pica Tarapacá (ca. 900-1450 DC). Actas del XVII Congreso Nacional de Arqueología Chilena. Ediciones Kultrún, Valdivia. 
MORAGAS, C., 1991. Antecedentes sobre un pukara y estructura de cumbre asociadas a un campo de geoglifos en la quebrada de Tarapacá, área de Mocha, I Región. Actas XII Congreso Nacional de Arqueología Chilena. Boletín del Museo Regional de La Araucanía, tomo 2: 25-39.

1995. Desarrollo de las comunidades prehispánicas del litoral Iquique-desembocadura río Loa. Actas XIII Congreso Nacional de Arqueología Chilena. Hombre y Desierto. Una perspectiva cultural, tomo 1: 65-80.

MUÑOZ, I., y J. CHACAMA, 2006. Complejidad social en las alturas de Arica: territorio, etnicidady vinculación con el Estado Inca. Ediciones Universidad de Tarapacá, Arica.

MURRA, J., 1975 [1972]. El control vertical de un máximo de pisos ecológicos en la economía de las sociedades andinas. En Visita a la provincia de León de Huanuco en 1562, Iñigo Ortiz de Zúñiga, J. V. Murra (Ed.), pp. 429-476. Universidad Nacional Hermilio Valdizán, Huanuco.

NIEMEYER, H., 1961. Excursiones a la sierra de Tarapacá. Arqueología, toponimia, botánica. Revista Universitaria XLVI: 97-114.

1989. El escenario geográfico. En Culturas de Chile, Prehistoria, J. Hidalgo, V. Schiappacasse, H. Niemeyer, C. Aldunate e I. Solimano (Eds.), pp. 1-12. Editorial Andrés Bello, Santiago.

NÚÑEZ, L.,1965. Prospección arqueológica en el norte de Chile. Estudios Arqueológicos 1: 9-36.

1966. Caserones-I, una aldea prehispánica del norte de Chile. Estudios Arqueológicos 2: 25-29.

1974. La agricultura prehistórica de los Andes Meridionales. Editorial Orbe. Universidad del Norte, Antofagasta.

1979. Emergencia y desintegración de la sociedad tarapaqueña: riqueza y pobreza de una quebrada del norte de Chile. Atenea 439: 163-213.

1982. Temprana emergencia de sedentarismo en el desierto chileno. Proyecto Caserones. Chungara 9: 80-122.

1984. El asentamiento Pircas: Nuevas evidencias de tempranas ocupaciones agrarias en el norte de Chile. Estudios Atacameños 7: 152-177.

PEARSALL, D., 1989. Paleoethnobotany. A Handbook of Proceadures. Academic Press, Inc., University of Missouri, Missouri.
SCHIAPPACASSE, V., V. CASTRO y H. NIEMEYER, 1989. Los desarrollos regionales en el Norte Grande. En Culturas de Chile. Prehistoria, J. Hidalgo, V. Schiappacasse, C. Aldunate e I. Solimano (Eds.), pp. 181-220. Editorial Andrés Bello, Santiago.

TRUE, D. L., 1980. Archaeological investigations in Northern Chile: Caserones. En Prehistoric Trails of Atacama: Arcaheology of Northern Chile, C. W. Meighan y D. L. True (Eds.), pp. 139-178. Monumenta Archaeologica 7, The Institute of Archaeology, University of California, Los Angeles.

URBINA, S., y L. ADÁN, 2006. Construcciones de uso público y su distribución en las quebradas tarapaqueñas durante el período Intermedio Tardío (900-1450 DC). Boletín de la Sociedad Chilena de Arqueología 39: 19-34.

URIBE, M., 2006. Arqueología de Pica-Tarapacá (norte de Chile): Reflexiones acerca de la complejidad y desigualdad social en los Andes Centro-Sur (1000-1450 DC). Estudios Atacameños 31:91114 .

URIBE, M., y L. ADÁN, 2005. Evolución social a través de la prehistoria tardía de Pica-Tarapacá (Norte Grande de Chile). Ponencia presentada en Primer Taller de Teoría Arqueológica en Chile, Santiago.

URIBE, M., L. SANHUEZA y F. BAHAMONDES, 2007. Acercamiento sistemático a la cerámica prehispánica tardía de Tarapacá, norte de Chile: sus valles interiores y costa desértica (950-1532 d.C.). Chungara 39:143-170.

VAN KESSEL, J., 1992. Tecnología aymara: un enfoque cultural. En La cosmovisión aymara, H. Van den Berg y N. Schiffers (Eds.). Biblioteca Andina, La Paz.

VALENZUELA, J., 2009. El material malacológico y el Complejo Cultural Pica-Tarapacá: uso social y simbolismo de las conchas en la prehistoria tardía (fase Camiña 1200-1450 DC). Memoria de título en Arqueología, Facultad de Ciencias Sociales, Universidad de Chile.

VIDAL, A., 2008. Informe de análisis de restos arqueobotánicos de Tarapacá Viejo (T-49), Región de Tarapacá. Proyecto FONDECYT 7060165.

VILLAGRÁN, C., y V. CASTRO, 2004. Ciencia indígena de los Andes del norte de Chile. Editorial Universitaria, Santiago.

VILLAGRÁN, C., V. CASTRO, G. SÁNCHEZ, F. HINOJOSA y C. LATORRE, 1999. La tradición altiplánica: estudio etnobotánico en los Andes de Iquique, Primera Región, Chile. Chungara 31: 81-186. 